\title{
HOMOLOGY OF AZUMAYA ALGEBRAS
}

\author{
G. CORTIÑAS AND C. WEIBEL
}

(Communicated by Maurice Auslander)

\begin{abstract}
If $R$ is a commutative $k$-algebra, any Azumaya $R$-algebra has the same Hochschild homology as $R$ does.
\end{abstract}

Let $R$ be a commutative ring. An Azumaya algebra over $R$ is a (noncommutative) $R$-algebra $A$ which is a finitely generated faithful projective $R$-module, such that the canonical map $A \otimes_{R} A^{\mathrm{op}} \rightarrow \operatorname{End}_{R}(A)$ is an isomorphism (equivalently $A \otimes_{R} F$ is a central simple $F$-algebra for every $R \rightarrow F$ with $F$ a field. (See [B, II.5, Example $14 \mathrm{ff}]$.) For the étale topology on $R$, an Azumaya algebra is locally a matrix algebra; if $A$ has rank $n^{2}$ there exists a faithfully flat étale $R$-algebra $S$ such that $A \otimes_{R} S \cong M_{n}(S)$ by [GB, 5.1]. Thus it is reasonable to expect Azumaya algebras to satisfy some kind of homological Morita invariance conditions, and our result says just that. Suppose that $k$ is a commutative subring and that we consider Hochschild homology $H H_{*}^{k}$ of $R$ and $A$ as $k$-algebras.

Main Theorem. If $A$ is an Azumaya algebra over a k-algebra $R$, then there are "reduced trace map" isomorphisms $H H_{*}^{k}(A) \stackrel{\cong}{\rightrightarrows} H H_{*}^{k}(R)$. If $A$ has rank $n^{2}$, the composition $H H_{*}^{k}(R) \rightarrow H H_{*}^{k}(A) \cong H H_{*}^{k}(R)$ induced by $R \subset A$ is multiplication by $n$.

For example, if $P$ is an f.g. faithful projective $R$-module then $A=\operatorname{End}_{R}(P)$ is Azumaya over $R$. In this case we recover the usual Morita invariance:

$$
H H_{*}^{k} \operatorname{End}_{R}(P) \cong H H_{*}^{k}(R) .
$$

Corollary. If $A$ is an Azumaya algebra of rank $n^{2}$ over $R$ and $1 / n \in R$, then $R \rightarrow A$ induces an isomorphism $H C_{*}(R) \cong H C_{*}(A)$.

If we take $k=R$, we get an improvement of a result of Larsen [L].

Received by the editors November 11, 1991 and, in revised form, August 24, 1992.

1991 Mathematics Subject Classification. Primary 16E40, 08G60.

Key words and phrases. Azumaya algebra, Hochschild homology.

The first author was supported by the Consejo Nacional de Investigaciones Cientificas y Técnicas de La Argentina. This author is indebted to Rutgers University for its hospitality during his CONICET sponsored stay.

The second author was partially supported by NSF grant DMS-8803497. 
Corollary. If $A$ is an Azumaya k-algebra, then $H H_{i}(A)=0$ for $i \neq 0$, and $A /[A, A] \cong k$. By the $S B I$ sequence, there is an isomorphism $H C_{*}(A) \cong$ $H C_{*}(k)$.

To prove our main theorem we need the following variation of Theorem 0.1 of [GW].

Étale Descent Theorem. Let $R \subset S$ be an étale extension of commutative $k$ algebras, and let $A$ be an $R$-algebra. Then the natural product is an isomorphism $H H_{*}^{k}(A) \otimes_{R} S \stackrel{\cong}{\Rightarrow} H H_{*}^{k}\left(A \otimes_{R} S\right)$.

The proof of this variant is the same (mutatis mutandis) as the proof in [GW]. Applying this to the Azumaya case at hand, and using the usual trace map for $A \otimes_{R} S \cong M_{n}(S)$ yields

$$
H H_{*}(A) \otimes_{R} S \cong H H_{*}\left(A \otimes_{R} S\right) \cong H H_{*}(S) .
$$

Another application of étale descent yields $H H_{*}(R) \otimes_{R} S \cong H H_{*}(S)$. We may therefore consider $H H_{*}(A)$ and $H H_{*}(R)$ as $R$-submodules of $H H_{*}(S)$. Once we prove that they are the same submodule, we can take the reduced trace map to be the composite $H H_{*}(A) \subset H H_{*}\left(A \otimes_{R} S\right) \cong H H_{*}(S)$; since the image lands in $H H_{*}(R)$, the trace map is independent of the choice of $S$.

If two submodules differ, they differ locally. Since locally every Azumaya algebra contains a maximal étale subalgebra (see [GB, 5.7]), and $H H_{*}(A[1 / t])=$ $H H_{*}(A) \otimes_{R} R[1 / t]$, the general case reduces to the special case in which $R \subset$ $S \subset A, A \otimes_{R} S \cong M_{n}(S)$ and $S$ is étale of rank $n$ over $R$.

This reduction being made, $S \subset A$ induces a map from $H H_{*}(S)$ to $H H_{*}(A)$ $\subset H H_{*}(S)$. If $x \in H H_{*}(R)$ and $s \in S$, this map sends $x \otimes s \in H H_{*}(R) \otimes_{R} S=$ $H H_{*}(S)$ to $x \otimes \operatorname{Tr}(s)$. Indeed, there is a commutative diagram:

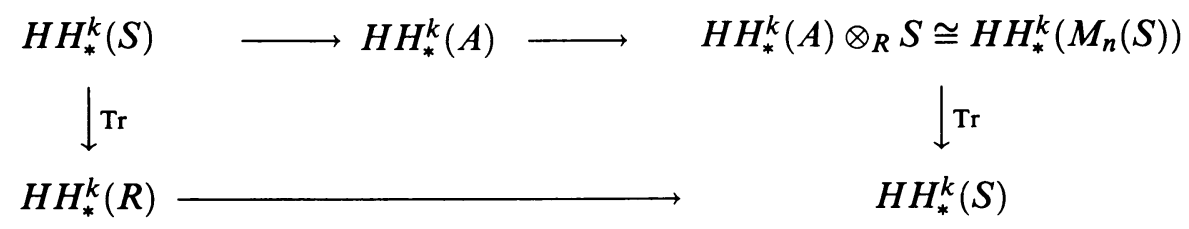

But $\operatorname{Tr}: S \rightarrow R$ is onto, so the image of $H H_{*}(S)$ is $H H_{*}(R)$. This proves that $H H_{*}(R) \subset H H_{*}(A)$ as $R$-submodules of $H H_{*}(S)$. Tensoring with $S$ makes this map an isomorphism, so by faithfully flat descent it must already be an isomorphism. This finishes the proof of our main theorem.

\section{ACKNOWLEDGMENTS}

The authors were inspired by Proposition 1.6 of [L]. We understand that Don Schack has independently proven the corresponding results for cohomology in [S].

\section{BIBLIOGRAPHY}

[B] N. Bourbaki, Commutative algebra, Hermann, Paris, 1972.

[GB] A. Grothendieck, Le groupe de Brauer. I, Dix Exposés sur la Cohomologie des Schemas, North-Holland, Amsterdam, 1966. 
[GW] S. Geller and C. Weibel, Étale descent for Hochschild and cyclic homology, Comment. Math. Helv. 66 (1991), 368-388.

[L] M. Larsen, Homology of maximal orders in central simple algebras, preprint, 1991.

[S] S. D. Schack, Bimodules, the Brauer group, Morita equivalence, and cohomology, J. Pure Appl. Algebra 80 (1992), 315-325.

Department of Mathematics, Rutgers University, New Brunswick, New Jersey 08903 Current address, G. Cortiñas: Department of Mathematics, Washington University, Campus Box 1146, St. Louis, Missouri 63130

E-mail address, G. Cortinas: cortinas@math.wustl.edu

E-mail address, C. Weibel: weibel@math.rutgers.edu 